\title{
Subbarrier fusion reactions of an aligned deformed nucleus
}

\author{
K. Hagino ${ }^{1}$ and S. Sakaguchi ${ }^{2}$ \\ 1 Department of Physics, Kyoto University, Kyoto 606-8502, Japan \\ 2 Department of Physics, Kyushu University, Fukuoka 819-0395, Japan
}

\begin{abstract}
We discuss heavy-ion fusion reactions of a well-deformed odd-mass nucleus at energies around the Coulomb barrier. To this end, we consider the ${ }^{16} \mathrm{O}+{ }^{165} \mathrm{Ho}$ reaction and take into account the effect of deformation of ${ }^{165} \mathrm{Ho}$ using the orientation average formula. We show that fusion cross sections are sensitive to magnetic substates of the target nucleus, and cross sections for the side collision, which are relevant to a synthesis of superheavy elements, may be enhanced by a factor of around two by aligning the deformed target nuclei. We also discuss the effect of alignment on the fusion barrier distribution. We find that, for a particular choice of alignment, the shape of barrier distribution becomes similar to a typical shape of barrier distribution for a deformed nucleus with a negative hexadecapole deformation parameter, $\beta_{4}$, even if the intrinsic $\beta_{4}$ is positive in the target nucleus.
\end{abstract}

\section{INTRODUCTION}

It has been well known that nuclear deformation of the colliding nuclei plays an important role in heavy-ion fusion reactions [1 [5]. For medium-heavy systems, such as ${ }^{16} \mathrm{O}+{ }^{154} \mathrm{Sm}$, fusion cross sections are largely enhanced at energies around the Coulomb barrier compared to a prediction of a simple potential model. This has been interpreted as a result of a distribution of the Coulomb barriers, that depend upon the orientation angle of the deformed target nucleus. Since fusion cross sections have an exponential dependence on the incident energy at energies below the Coulomb barrier, fusion cross sections can be enhanced by orders of magnitude due to the contribution of the configurations for which the Coulomb barrier is lowered than the original barrier. The barrier distribution has been investigated experimentally for several systems [2, 6, 7], and this picture has been well established by now.

The nuclear deformation plays an important role also in fusion reactions in massive systems, that is, those used to synthesize superheavy nuclei. For prolately deformed nuclei, a compact configuration is realized at the touching point when a projectile nucleus approaches from the direction of the shorter axis of the target nucleus, that is, the side collision. This makes it a big advantage to overcome an inner barrier and form a compound nucleus 8 14]. The notion of compactness has recently been confirmed experimentally by comparing the measured barrier distribution and the excitation function of evaporation residue cross sections for the ${ }^{48} \mathrm{Ca}+{ }^{248} \mathrm{Cm}$ system [15]. Moreover, employing the concept of the compactness, the so called hugging fusion was proposed for fusion between deformed nuclei with negative hexadecapole deformation, such as ${ }^{150} \mathrm{Nd}+{ }^{150} \mathrm{Nd}$, for which the touching configuration is compact when the symmetry axis of each nucleus is perpendicular to each other [16]. (Notice, however, that the effective inner barrier will be considerably high for such symmetric systems [17] and it would be extremely difficult to synthesize superheavy elements with hugging fusion.)
In many experiments for fusion of a deformed nucleus, an even-even nucleus has been chosen as a target nucleus, partly because the ground state has zero spin and thus the theoretical treatment is easy. However, a finite spin of odd-mass nuclei may bring an interesting insight into dynamics of heavy-ion fusion reactions [18] (see also Ref. [19]). As a matter of fact, fusion of an aligned/polarized nucleus has been theoretically investigated in the 1980s and 1990s and it has been demonstrated that fusion cross sections are significantly altered by aligning the odd-mass nuclei $20-24]$. Notice that this effect would be important in fusion in astrophysical environments under a strong magnetic field, which leads to a natural polarization of colliding nuclei. Experimentally, fusion of an aligned light nucleus, ${ }^{23} \mathrm{Na}$, has been measured 25 27]. For heavier deformed nuclei, a measurement of fusion cross sections for the ${ }^{16} \mathrm{O}+{ }^{165} \mathrm{Ho}$ system was planned [24], even though the actual experiment has not yet been performed so far [28].

In this paper, we revisit the problem of fusion of an aligned deformed nucleus. In the previous studies, the effect of alignment was discussed only in terms of fusion cross sections as well as tensor analyzing powers, and the effect on fusion barrier distributions has yet to be investigated. Notice that the shape of fusion barrier distribution is sensitive to the details of nuclear deformation, and one may gain a deeper insight into the reaction dynamics of a deformed nucleus by analyzing the fusion barrier distributions. Moreover, the effect of alignment has never been discussed in connection to fusion for superheavy elements, which may be important for future experiments to synthesize new superheavy elements. Given the new experiment for the barrier distribution for systems relevant to superheavy elements [15], we consider that it is timely to revisit this problem now.

The paper is organized as follows. In Sec. II, we summarize the theoretical framework to describe fusion cross sections for an aligned deformed target. To this end, we consider fusion of a well-deformed nucleus, for which fusion cross sections are approximately given as an average of fusion cross sections for fixed orientation angles. In Sec. III, we apply the formula to the ${ }^{16} \mathrm{O}+{ }^{165} \mathrm{Ho}$ reac- 
tion, and discuss the effect of alignment on the fusion cross sections and the fusion barrier distribution. We also discuss an implication of the effect of alignment for fusion for superheavy nuclei. We then summarize the paper in Sec. IV.

\section{FUSION CROSS SECTIONS FOR AN ALIGNED DEFORMED TARGET}

We consider fusion between an inert projectile and a well-deformed target nucleus. In this case, the relative motion between the colliding nuclei couples to the rotational motion of the deformed target. We take a rigid rotor model to describe the wave functions for the ground state rotational band of the target nucleus. That is, for the state with the angular momentum $I$ and its $z$ component $M$, the wave function reads [29].

$$
\begin{aligned}
\Psi_{I K M}(\Omega)= & \sqrt{\frac{2 I+1}{16 \pi^{2}\left(1+\delta_{K, 0}\right)}}\left(D_{M K}^{I}(\Omega) \phi_{K}(\xi)\right. \\
& \left.+(-1)^{I+K} D_{M-K}^{I}(\Omega) \phi_{\bar{K}}(\xi)\right),
\end{aligned}
$$

where $K$ is the $K$-quantum number, that is, the projection of the angular momentum on the body-fixed frame. Here, we have assumed that the deformed nucleus has axially symmetric shape so that the $K$-quantum number is conserved. $D_{M K}^{I}(\Omega)$ is the Wigner D-function, in which $\Omega=(\phi, \theta, \chi)$ denotes the Euler angles. $\phi_{K}(\xi)$ is the intrinsic wave function, where $\xi$ is the intrinsic coordinate, and $\phi_{\bar{K}}$ is the time-reverse of $\phi_{K}$.

To simplify the angular momentum coupling, we employ the iso-centrifugal approximation [3]. In this approximation, one transforms the whole system to the rotating frame where the $z$ axis is along the direction of the relative motion, $\boldsymbol{r}$, at every instant. The interaction between the projectile and the target nuclei in this approximation then reads [3, 30],

$$
V(r, \theta)=V_{N}(r, \theta)+V_{C}(r, \theta),
$$

with the nuclear potential given by,

$$
V_{N}(r, \theta)=-\frac{V_{0}}{1+\exp \left[\left(r-R_{0}-R_{T} \sum_{\lambda} \beta_{\lambda} Y_{\lambda 0}(\theta)\right) / a\right]},
$$

and the Coulomb potential given by,

$$
\begin{aligned}
V_{C}(r, \theta)= & \frac{Z_{P} Z_{T} e^{2}}{r}\left(1+\frac{3}{5} \frac{R_{T}^{2}}{r^{2}}\left(\beta_{2}+\frac{2}{7} \sqrt{\frac{5}{\pi}} \beta^{2}\right) Y_{20}(\theta)\right. \\
& \left.+\frac{3}{9} \frac{R_{T}^{4}}{r^{4}}\left(\beta_{4}+\frac{9}{7} \beta^{2}\right) Y_{40}(\theta)\right) .
\end{aligned}
$$

Here, $\beta_{\lambda}$ are the deformation parameters of the deformed target and $\theta$ denotes the angle between the symmetry axis of the deformed target and the relative coordinate, $\boldsymbol{r}$. For a prolately deformed target, $\theta=0(\theta=\pi / 2)$ corresponds to the case where the projectile nucleus approaches from the longer (shorter) axis of the target and refers to as the tip (side) collision. $R_{T}$ and $Z_{T}$ are the radius and the charge number of the target nucleus, respectively, and $Z_{P}$ is the charge number of the projectile nucleus. Here, we have assumed a Woods-Saxon shape for the nuclear potential, and expand the Coulomb potential up to the second order of $\beta_{2}$ and the first order of $\beta_{4}$. Notice that in the iso-centrifugal approximation the value of $M$ is conserved during the reaction, since the coupling potential, Eq. (2), does not change the $z$ component of the angular momentum [3, 31]. The fusion cross sections can thus be labeled with $M$.

In addition to the iso-centrifugal approximation, we take the sudden tunneling approximation by setting the rotational energy of the target nucleus to be zero. In this approximation, together with the iso-centrifugal approximation, fusion cross sections are given as a weighted average of cross sections for fixed values of the angle $\theta$, with the weight factors given by the square of the ground state wave function of the target nucleus [3, 20, 32 34]. That is, fusion cross sections for a magnetic substate $M$ read,

$$
\begin{aligned}
\sigma_{\text {fus }}^{(M)}(E)= & \int d \Omega\left|\Psi_{I_{0} K_{0} M}(\Omega)\right|^{2} \sigma_{\text {fus }}(\theta), \\
= & \int_{0}^{2 \pi} d \phi \int_{0}^{\pi} \sin \theta d \theta \int_{0}^{2 \pi} d \chi \\
& \times\left|\Psi_{I_{0} K_{0} M}(\Omega)\right|^{2} \sigma_{\text {fus }}(\theta),
\end{aligned}
$$

where $I_{0}$ and $K_{0}$ are the value of $I$ and $K$ for the ground state, and $\sigma_{\text {fus }}(\theta)$ is the fusion cross section evaluated with a potential given by Eq. (2) for a fixed value of $\theta$. Notice that $M$ is a projection of the angular momentum on the $z$-axis, which coincides with the beam direction at the initial stage of reaction. That is, the quantization axis for the ground state spin of the target nucleus is in the beam direction in this formula. Since the integrals of the $\phi$ and $\chi$ are trivial in Eq. (6), one finally obtains,

$$
\begin{aligned}
\sigma_{\text {fus }}^{(M)}(E)= & \frac{2 I_{0}+1}{2} \int_{0}^{\pi / 2} \sin \theta d \theta \\
& \times\left(\left|d_{M K_{0}}^{I_{0}}(\theta)\right|^{2}+\left|d_{M-K_{0}}^{I_{0}}(\theta)\right|^{2}\right) \sigma_{\text {fus }}(\theta)(7)
\end{aligned}
$$

which coincides with Eq. (19) in Ref. 24] (see also Ref. [35]). Here we have used the relation $\sigma_{\text {fus }}(\theta)=$ $\sigma_{\text {fus }}(\pi-\theta)$, which is valid for deformed nuclei with a reflection symmetric shape. Notice that, using the relation $\sum_{M}\left|d_{M K}^{I}(\theta)\right|^{2}=1$, fusion cross sections for the unpolarized target reads

$$
\begin{aligned}
\sigma_{\text {fus }}^{(\text {unpol })}(E) & =\frac{1}{2 I_{0}+1} \sum_{M} \sigma_{\text {fus }}^{(M)}(E), \\
& =\int_{0}^{\pi / 2} \sin \theta d \theta \sigma_{\text {fus }}(\theta),
\end{aligned}
$$

which is identical to the formula for even-even deformed nuclei [3]. 


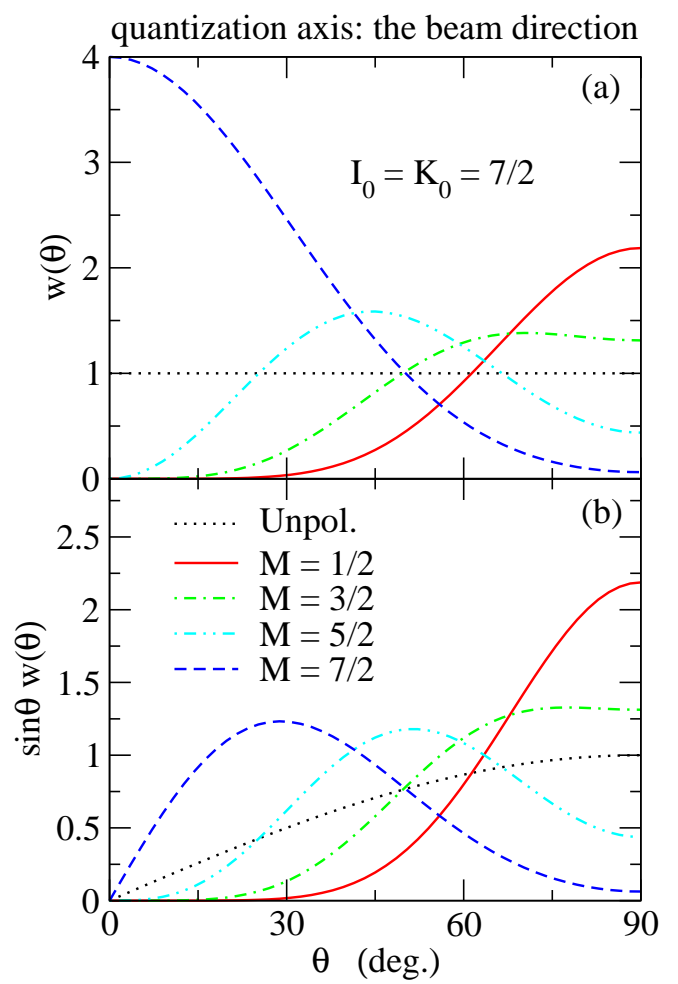

FIG. 1: The weight factor for fusion cross sections of a deformed target nucleus with the ground state spin and parity of $I_{0}^{\pi}=7 / 2^{-}$and the $K$ quantum number of $K_{0}=7 / 2$. It is plotted as a function of the angle between the symmetry axis of the target and the relative coordinate between the projectile and the target. The quantization axis is set to be parallel to the beam direction. The upper and the lower panels show the weight factor without and with the factor $\sin \theta$. The solid, the dot-dashed, the dot-dot-dashed, and the dashed lines are for $M=1 / 2,3 / 2,5 / 2$, and $7 / 2$, respectively, where $M$ is the projection of the ground state spin of the target onto the quantization axis. The weight factor for the unpolarized case is also shown by the dotted lines.

Fusion cross sections for another direction of the quantization axis can also be computed by expanding the quantized state, $|I \tilde{M}\rangle$, with the eigenstates of $I_{z}$ as,

$$
|I \tilde{M}\rangle=\sum_{M} c_{M}|I M\rangle
$$

Notice that the absolute value of the expansion coefficient, $c_{M}$, is actually given by

$$
\left|c_{M}\right|=\left|d_{\tilde{M} M}^{I}\left(\theta_{a}\right)\right|,
$$

where $\theta_{a}$ is the angle between the quantization axis and the $z$-axis. Fusion cross sections are then given by,

$$
\sigma_{\text {fus }}^{(\tilde{M})}(E)=\sum_{M}\left|c_{M}\right|^{2} \sigma_{\text {fus }}^{(M)}(E)
$$

where $\sigma_{\text {fus }}^{(M)}(E)$ is the fusion cross section when the quantization axis is taken to be the $z$-axis, given by Eq. (6).

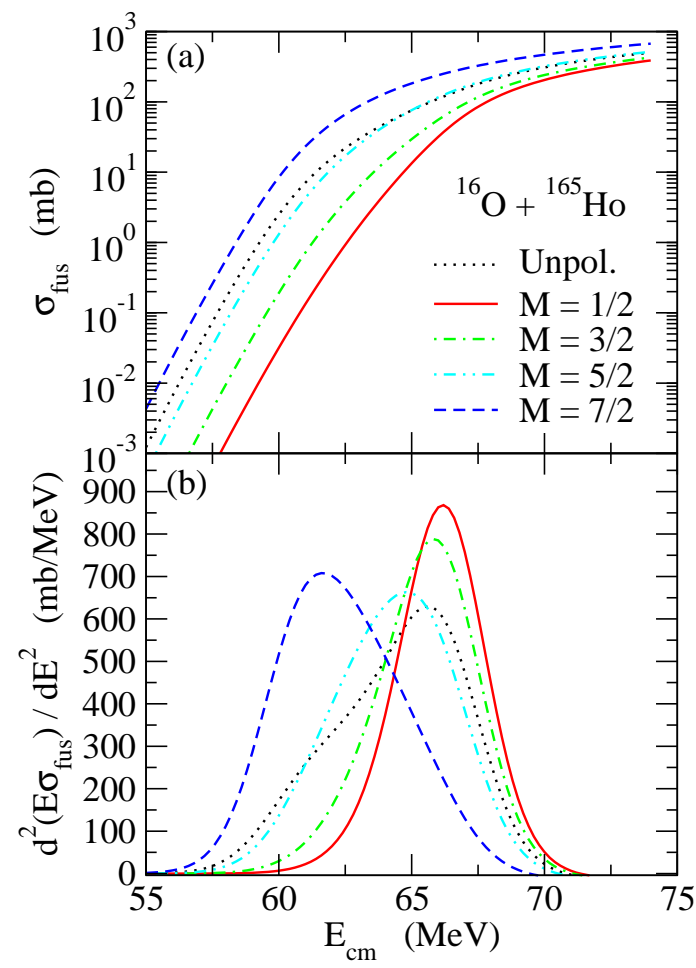

FIG. 2: Fusion cross sections (the upper panel) and the fusion barrier distribution (the lower panel) for the ${ }^{16} \mathrm{O}+{ }^{165} \mathrm{Ho}$ reaction. The meaning of each line is the same as in Fig. 1.

\section{APPLICATION TO THE ${ }^{16} \mathrm{O}+{ }^{165} \mathrm{Ho}$ SYSTEM}

Let us now apply the formulas in the previous section to the ${ }^{16} \mathrm{O}+{ }^{165} \mathrm{Ho}$ system and discuss the effect of alignment of the target nucleus, ${ }^{165} \mathrm{Ho}$, that is a well deformed nucleus with the ground state spin and parity of $I_{0}=7 / 2^{-}$. The ground state rotational band has $K_{0}^{\pi}=7 / 2^{-}$. Notice that this nucleus has an ideal property as a material of the spin-aligned target. That is, due to its extremely strong hyperfine field, a large spin alignment of up to $80-90 \%$ of the maximum theoretical value can be achieved by cooling down a single crystal of Ho metal 37 42].

We first take the quantization axis to be parallel to the beam direction. Figure 1 shows the weight factor in the fusion cross sections (see Eq. (77)),

$$
w_{M}(\theta) \equiv \frac{2 I_{0}+1}{2}\left(\left|d_{M K_{0}}^{I_{0}}(\theta)\right|^{2}+\left|d_{M-K_{0}}^{I_{0}}(\theta)\right|^{2}\right),
$$

without (the upper panel) and with (the lower panel) the statistical factor of $\sin \theta$. For comparison, the weight factor for the unpolarized case, $w(\theta)=1$, (see Eq. (9) ) is also shown. One can see that, whereas the distribution is isotropic for the unpolarized case, the side (the tip) collision is more emphasized for $M=1 / 2(M=7 / 2)$.

In fusion reactions to synthesize superheavy elements, the side collision around $\theta \sim \pi / 2$ contributes predominantly [14, 15]. The figure implies that evaporation 


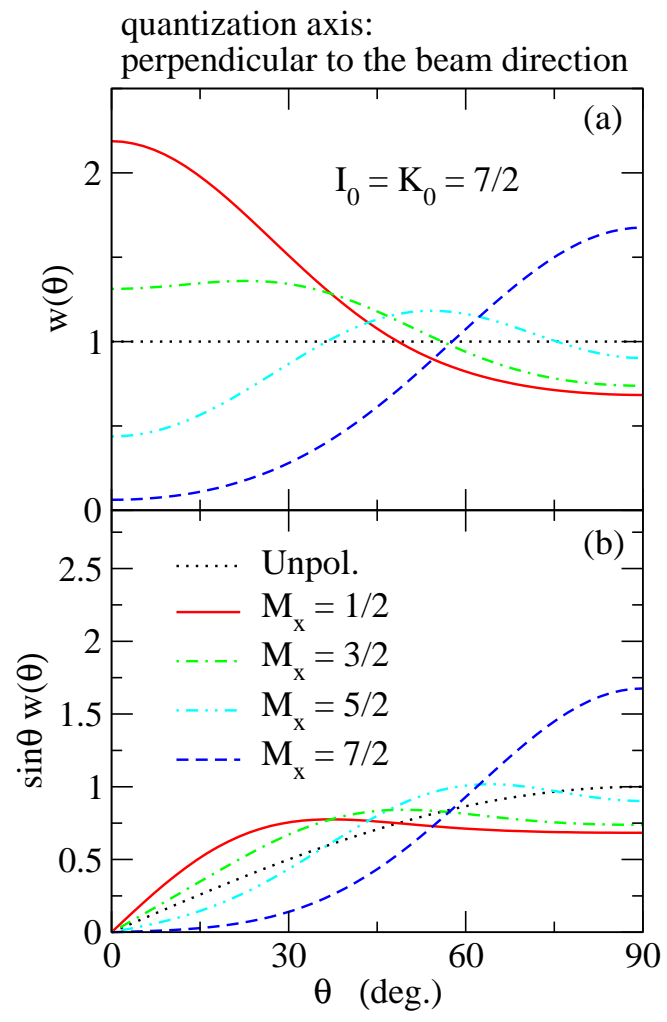

FIG. 3: Same as Fig. 1, but for the case where the quantization axis is perpendicular to the beam direction.

residue cross sections of superheavy nuclei could be increased by a factor of around two as compared to the unpolarized case, if the target nucleus could be selectively prepared with $M= \pm 1 / 2$. In the synthesis of superheavy elements with extremely low cross sections as well as in fusion reaction with low intensity radioactive-ion beams, it is critically important to reduce beam times by making all possible efforts. The enhancement by a factor of two suggested by this calculation indicates that a spin alignment can be used for such purposes, even though a spin-aligned target which is applicable to fusion measurements will have to be developed.

Fusion cross sections for the ${ }^{16} \mathrm{O}+{ }^{165} \mathrm{Ho}$ are shown in the upper panel of Fig. 2. To this end, we take the Woods-Saxon form for the internuclear potential, with the depth, the radius, and the diffuseness parameters of $V_{0}=104 \mathrm{MeV}, r_{0}=1.15 \mathrm{fm}$, and $a=0.63 \mathrm{fm}$, respectively. This potential yields a similar barrier height as that with the Akyüz-Winther potential [43]. For the deformation parameters, we follow Ref. [36] and take $\beta_{2}=0.284$ and $\beta_{4}=0.020$, with the radius parameter of $R_{T}=1.2 A_{T}^{1 / 3} \mathrm{fm}$. The excitation of ${ }^{16} \mathrm{O}$ is taken into account only through the potential renormalization [3, 44], and is not explicitly included in the calculations. The figure corresponds well to Fig. 3 in Ref. 24]. Since the tip collision has a lower Coulomb barrier than the side collision, fusion cross sections for $M=7 / 2$ are much more enhanced as compared to those for $M=1 / 2$, reflecting

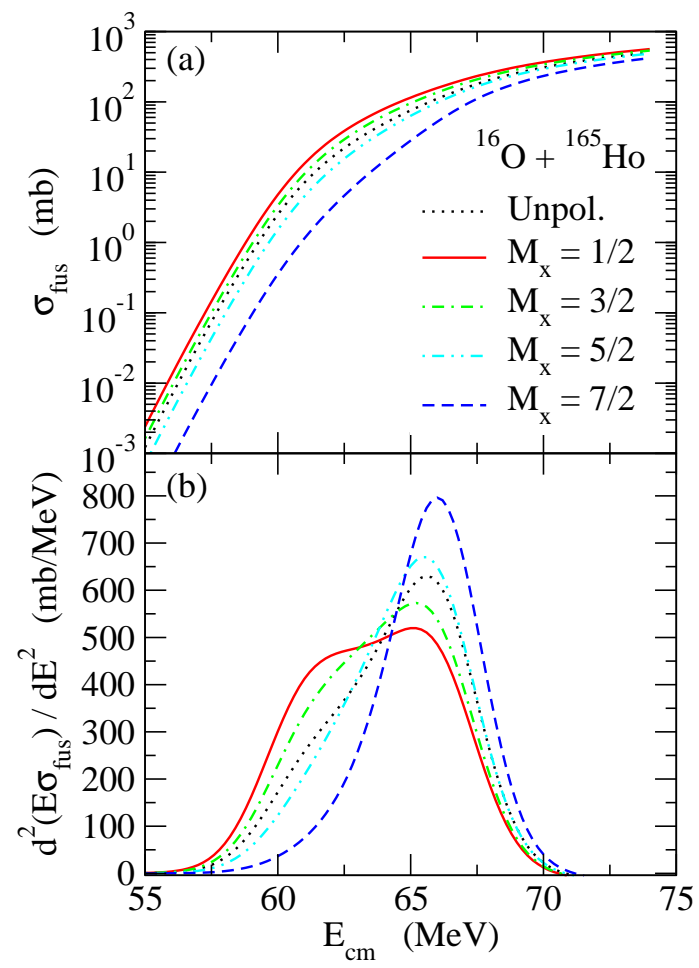

FIG. 4: Same as Fig. 2, but for the case where the quantization axis is perpendicular to the beam direction.

the angle dependence of the weight factor as shown in Fig. 1.

This fact can be seen more transparently in the fusion barrier distributions shown in the lower panel of Fig. 2. Here, the fusion barrier distribution, $D_{\text {fus }}(E)$, is defined as the second energy derivative of $E \sigma_{\text {fus }}(E)[2,[6]$, that is,

$$
D_{\text {fus }}(E)=\frac{d^{2}\left(E \sigma_{\text {fus }}\right)}{d E^{2}} .
$$

Here, we evaluate the barrier distributions using the point difference formula 2] with the energy step of $\Delta E=$ $2 \mathrm{MeV}$. One can see that the shape of barrier distribution is considerably altered by the alignment, and moreover it is sensitive to the value of $M$, again by reflecting the angle dependence of the weight factor.

Let us next discuss the case where the quantization axis of the ground state spin of the target nucleus is perpendicular to the beam direction, that is, the $x$ axis. Fig. 3 shows the weight factor, $\tilde{w}_{M_{x}}(\theta)$,

$$
\tilde{w}_{M_{x}}(\theta)=\sum_{M}\left|d_{M_{x} M}^{I_{0}}(-\pi / 2)\right|^{2} w_{M}(\theta)
$$

where $w(\theta)$ is the weight factor given by Eq. (13). Here we have used the notation $M_{x}$ to denote the magnetic substates in this case, in order to distinguish them from those in the case where the quantization axis is along the beam axis. One can see that the role of $M=1 / 2$ and $M=7 / 2$ is inverted from the case where the quantization 


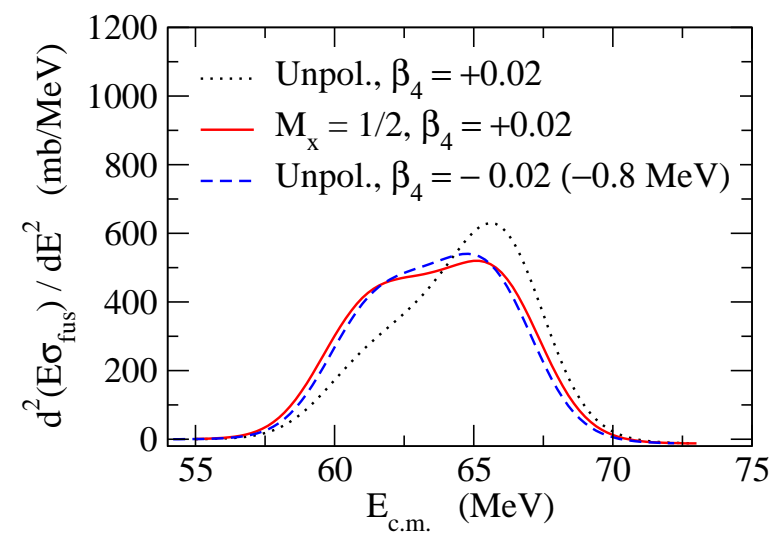

FIG. 5: Comparison of the fusion barrier distributions for the ${ }^{16} \mathrm{O}+{ }^{165}$ Ho reaction obtained with several schemes. The meaning of the dotted and the solid lines is the same as in Fig. 4 (b), that is, the dotted line denotes the barrier distribution for the unpolarized case while the solid line shows the result for $M_{x}=1 / 2$ with the quantization axis being perpendicular to the beam direction. The dashed line shows the barrier distribution for the unpolarized case, but by changing the sign of the hexadecapole deformation parameter of ${ }^{165}$ Ho. To facilitate the comparison, the dashed line is shifted in energy by $-0.8 \mathrm{MeV}$.

axis is parallel to the beam axis (see Fig. 1). That is, the tip (the side) collision is more emphasized for $M_{x}=1 / 2$ $\left(M_{x}=7 / 2\right)$, that is opposite to Fig. 1 . For $M_{x}=7 / 2$, even though the weight at $\theta=\pi / 2$ is somewhat reduced compared to the weight factor for $M=1 / 2$ shown in Fig. 1 , it is still significantly larger than the weight factor for the unpolarized case, by a factor of about 1.5 . This would be an important implication for evaporation residue cross sections for superheavy nuclei.

The fusion cross sections and the fusion barrier distributions are shown in Fig. 4. The fusion cross sections for $M_{x}=7 / 2$ are considerably smaller than those for the other values of $M_{x}$, because this configuration contains much smaller component of the tip collision $(\theta \sim 0)$, which has the lowest Coulomb barrier, compared to the other configurations (see Fig. 3). As in the case where the quantization axis is parallel to the beam axis, one can see that the shape of barrier distribution depends sensitively on the value of $M_{x}$. Interestingly, the shape of barrier distribution for $M_{x}=1 / 2$ is a typical one for an even-even deformed nucleus with $\beta_{2}>0$ and $\beta_{4}<0$ [2, 7]. To demonstrate this, Fig. 5 compares the barrier distribution for $M_{x}=1 / 2$ (the solid line) with that for the unpolarized case obtained by inverting the sign of $\beta_{4}$ (the dashed line). To facilitate the discussion, the solid line is shifted in energy by $-0.8 \mathrm{MeV}$. For a comparison, the figure also shows the barrier distribution for the un- polarized case with the positive sign of $\beta_{4}$ (the dotted line). In addition to the well known fact that the shape of barrier distribution is sensitive to the sign of $\beta_{4}$ [2, 7], one can see that the solid line is indeed similar to the dashed line. This may suggest that the hexadecapole deformation can be effectively changed rather arbitrarily in heavy-ion fusion reactions by appropriately aligning an odd-mass target, even though the intrinsic hexadecapole deformation itself remains the same.

\section{SUMMARY}

We have discussed the role of alignment in heavy-ion subbarrier fusion reactions of a well-deformed odd-mass nucleus. Such nucleus has a finite spin in the ground state, and fusion cross sections differ depending on how the nucleus is polarized. We have in particular considered the ${ }^{16} \mathrm{O}+{ }^{165} \mathrm{Ho}$ system, and employed the iso-centrifugal and the sudden tunneling approximations. With these approximations, the fusion cross sections are given as a weighted average of orientation dependent cross sections, in which the weight factor is given in terms of the ground state wave function of the target nucleus for each quantum number $M, M$ being the projection of the initial spin of the deformed target nucleus on to the direction of the beam axis. We have shown that the fusion cross sections and the shape of barrier distribution is sensitive to the magnetic substate of the target nucleus. In particular, whereas $\beta_{4}$ is positive in ${ }^{165} \mathrm{Ho}$, the shape of barrier distribution becomes similar to a typical one for an even-even deformed nucleus with a negative value of $\beta_{4}$, when the odd-mass target is aligned along the axis perpendicular to the beam axis. This may imply that one can control the hexadecapole deformation in subbarrier fusion reactions by aligning deformed target nuclei. We have also pointed out that fusion cross sections for the side collision can be enhanced by a factor of around 2, by selectively choosing a particular value of a magnetic substate. This would be a good advantage for synthesizing superheavy elements, for which the side collision predominantly contributes. Moreover, use of a spin-aligned target would enable one to control the orientation of the deformed nuclei and to investigate the dynamics of fusion reactions by decomposing tip and side contributions.

\section{Acknowledgments}

We thank Phil Walker for useful conversations. This work was supported by JSPS KAKENHI Grant Number JP19K03861.
[1] A.B. Balantekin and N. Takigawa, Rev. Mod. Phys. 70, 77 (1998).
[2] M. Dasgupta, D.J. Hinde, N. Rowley, and A.M. Stefanini, 
Annu. Rev. Nucl. Part. Sci. 48, 401 (1998).

[3] K. Hagino and N. Takigawa, Prog. Theor. Phys. 128, 1061 (2012).

[4] B.B. Back, H. Esbensen, C.L. Jiang, and K.E. Rehm, Rev. Mod. Phys. 86, 317 (2014).

[5] G. Montagnoli and A.M. Stefanini, Eur. Phys. J. A53, 169 (2017).

[6] N. Rowley, G.R. Satchler, and P.H. Stelson, Phys. Lett. B254, 25 (1991).

[7] J.R. Leigh, M. Dasgupta, D.J. Hinde, J.C. Mein, C.R. Morton, R.C. Lemmon, J.P. Lestone, J.O. Newton, H. Timmers, J.X. Wei, and N. Rowley, Phys. Rev. C52, 3151 (1995).

[8] D.J. Hinde, D.Y. Jeung, E. Prasad, A. Wakhle, M. Dasgupta, M. Evers, D.H. Luong, R. du Rietz, C. Simenel, E.C. Simpson, and E. Williams, Phys. Rev. C97, 024616 (2018).

[9] D.J. Hinde, M. Dasgupta, J.R. Leigh, J.P. Lestone, J.C. Mein, C.R. Morton, J.O. Newton, and H. Timmers, Phys. Rev. Lett. 74, 1295 (1995).

[10] D.J. Hinde, M. Dasgupta, J.R. Leigh, J.C. Mein, C.R. Morton, J.O. Newton, and H. Timmers, Phys. Rev. C53, 1290 (1996).

[11] K. Nishio, H. Ikezoe, S. Mitsuoka, I. Nishinaka, Y. Nagame, Y. Watanabe, T. Ohtsuki, K. Hirose, and S. Hofmann, Phys. Rev. C77, 064607 (2008).

[12] K. Nishio, H. Ikezoe, S. Mitsuoka, and J. Lu, Phys. Rev. C62, 014602 (2000).

[13] N. Rowley, N. Grar, and M. Trotta, Phys. Rev. C76, 044612 (2007).

[14] K. Hagino, Phys. Rev. C98, 014607 (2018).

[15] T. Tanaka, Y. Narikiyo, K. Morita, K. Fujita, D. Kaji, K. Morimoto, S. Yamaki, Y. Wakabayashi, K. Tanaka, M. Takeyama, A. Yoneda, H. Haba, Y. Komori, S. Yanou, B.J.-P. Gall, Z. Asfari, H. Faure, H. Hasebe, M. Huang, J. Kanaya, M. Murakami, A. Yoshida, T. Yamaguchi, F. Tokanai, T. Yoshida, S. Yamamoto, Y. Yamano, K. Watanabe, S. Ishizawa, M. Asai, R. Aono, S. Goto, K. Katori, and K. Hagino, J. Phys. Soc. Jpn. 87, 014201 (2018).

[16] A. Iwamoto, P. Möller, J.R. Nix, and H. Sagawa, Nucl. Phys. A596, 329 (1996).

[17] T. Cap, K. Siwek-Wilczynska, and J. Wilczynski, Phys. Lett. B736, 478 (2014).

[18] G. Colucci, G. Montagnoli, A.M. Stefanini, K. Hagino, A. Caciolli, P. Colovic, L. Corradi, E. Fioretto, F. Galtarossa, A. Goasdu, J. Grebosz, M. Mazzocco, D. Montanari, C. Parascandolo, F. Scarlassara, M. Siciliano, E. Strano, S. Szilner and N. Vukman, Eur. Phys. J. A55,111 (2019).

[19] E. Piasecki, M. Kowalczyk, S. Yusa, A. Trzcinska, and K. Hagino, Phys. Rev. C100, 014616 (2019).

[20] R.G. Stokstad and E.E. Gross, Phys. Rev. C23, 281 (1981).

[21] P. Jacobs and U. Smilansky, Nucl. Phys. A438, 536 (1985).
[22] P. Fröbrich and G. Grawert, Nucl. Phys. A451, 338 (1986).

[23] I. Martel, J. Gómez-Camacho, and M.V. Andrés, Phys. Lett. B279, 218 (1992).

[24] J.A. Christley, R.C. Johnson, and I.J. Thompson, J. of Phys. G20, 169 (1994).

[25] R. Butsch, H. Jänsch, D. Krämer, K-H. Möbius, W. Ott, E. Steffens, G. Tungate, A. Weller, K. Becker, K. Blatt, H. Leucker, W. Luck, D. Fick, and P. Paul, Phys. Rev. C36, 1351 (1987).

[26] H. Reich, K. Becker, M. Detje, W. Korsch, H. Leucker, Z. Moroz, K. Rusek, H.-G. Völk, W. Widdra, and D. Fick, Nucl. Phys. A576, 409 (1994).

[27] D. Fick, G. Grawert, and I.M. Turkiewicz, Phys. Rep. 214, 1 (1992).

[28] P.M Walker, private communications.

[29] D.J. Rowe, Nuclear Collective Motion (Methuen, London, 1970).

[30] K. Hagino, N. Rowley, and A.T. Kruppa, Comp. Phys. Comm. 123, 143 (1999).

[31] K. Hagino, N. Takigawa, A.B. Balantekin, and J.R. Bennett, Phys. Rev. C52, 286 (1995).

[32] N. Takigawa, Y. Alhassid, and A.B. Balantekin, Phys. Rev. C45, 1850 (1992).

[33] M.A. Nagarajan, A.B. Balantekin, and N. Takigawa, Phys. Rev. C34, 894 (1986).

[34] C.Y. Wong, Phys. Rev. Lett. 31, 766 (1973).

[35] D.J. Hinde, N. Rowley, M. Dasgupta, R.D. Butt, C.R. Morton, and A. Mukherjee, Phys. Rev. C68, 044606 (2003).

[36] P. Möller, A.J. Sierk, T. Ichikawa, and H. Sagawa, At. Data and Nucl. Data Tables, 109-110, 1 (2016).

[37] H. Postma, M. C. E. Smulders and W. J. Huiskamp, Physica27, 245 (1961).

[38] R. Wagner, P. D. Miller and T. Tamura, Phys. Rev.139 B29 (1965).

[39] M. A. Kelly, B. L. Berman, R. L. Bramblett and S. C. Fults, Phys. Rev. 1791194 (1969).

[40] N. J. Stone and H. Postma, Low-temperature Nuclear Orientation, edited by N. J. Stone and H. Postma (NorthHolland, Amsterdam, 1968).

[41] J. E. Koster, C. R. Gould, D. G. Haase and N. R. Roberson, Nucl. Instr. Meth. Phys. Res. A 313, 464 (1992).

[42] P. R. Huffman, N. R. Roberson, W. S. Wilburn, C. R. Gould, D. G. Haase, C. D. Keith, B. W. Raichle, M. L. Seely and J. R. Walston, Phys. Rev. Lett. 76, 4681 (1996).

[43] O. Akyüz and A. Winther, in Nuclear Structure and Heavy-Ion Physics, Proc. of the Int. School of Physics, "Enrico Fermi", Course LXXVII, Varenna, 1979, ed. by R.A. Broglia, C.H. Dasso and R. Richi (North-Holland, Amsterdam, 1981).

[44] N. Takigawa, K. Hagino, M. Abe, and A.B. Balantekin, Phys. Rev. C49, 2630 (1994). 